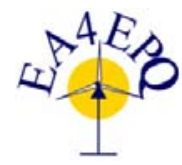

International Conference on Renewable Energies and Power Quality (ICREPQ’13) Bilbao (Spain), $20^{\text {th }}$ to $22^{\text {th }}$ March, 2013

Renewable Energy and Power Qualiuy. Fournal (RE\&PQJ)

ISSN 2172-038 X, No.11, March 2013

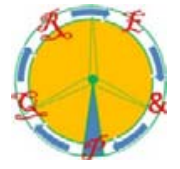

\title{
Rural Smart Grids: planning, operation and control review
}

\author{
I. Zubia ${ }^{1}$, I. Arrambide ${ }^{1}$, O.Azurza ${ }^{1}$ P.M. García ${ }^{1}$ and J.J. Ugartemendia ${ }^{1}$ \\ ${ }^{1}$ Department of Electrical Engineering \\ UPV/EHU University of the Basque Country \\ EUP, Plaza Europa 1, 20.018 Donostia-San Sebastián (Spain) \\ Phone/Fax number + 34943 017238/+ 34943 017130, e-mail: itziar.zubia@ehu.es, inaki.arrambide@ehu.es \\ olatz.azurza@ehu.es, pedro.garcia@ehu.es, juanjo.ugartemendia@ehu.es
}

\begin{abstract}
Rural Smart Grids enables the incorporation of distributed generation as while as the development of the rural communities. In this paper Smart Grid characteristics are presented and challenges of its integration in the network are analysed. Differential characteristics of Rural Smart Grids are summarized and areas that should be investigated to achieve their high scale implementation are pointed out.
\end{abstract}

\section{Key words}

Smart Grid, Distribution network, Rural electrification Simulation, Control.

\section{Introduction}

When the term Smart Grid is used, it refers to the entire electrical system, including generation, transmission, distribution and customers, but also operation, market and service providers that make the grid "smarter" [1]. The distribution system (generally either $25 \mathrm{kV}$ or $15 \mathrm{kV}$ ) is the largest and most complex part of the entire electrical system. For some large urban utilities that also have transmission and generation facilities, is sometimes the most neglected part of the system regarding its planning, operations and maintenance. For the smaller rural utilities, it receives much more attention. In addition, some feel that for rural parts of the distribution system, Smart Grid technologies cannot be utilized. If we speak today of Smart Cities, why not apply it to rural?

Rural Smart Grid consists of applying innovative solutions to the electrical generation in the countryside. Many of these networks are being promoted by Agriculture and Environment Departments or the rural communities. The idea is to deploy smart grids to harness energy that can be generated in the territory, improve power quality and energy efficiency. At the same time, they are used as a strategy for the growth and the creation of social and agroindustrial opportunities in rural areas, reinvesting in a benefit to the whole society.
Faced with Rural Micro-grids that are being implemented in developing countries, Rural Smart Grids are developed for years in the United States, related to rural electrification and using energy that can be generated in the territory.

In Europe, the European Technology Platform for the Electricity Networks of the Future, also called SmartGrids ETP, committed to the development of smart grids as a basic infrastructure for regional growth and create wealth, to be able to achieve a $35 \%$ renewable energy generation distributed through the territory in 2020 and the total energy decarbonisation by 2050 [2]. In Germany, the cooperative model is being successfully utilized for renewable energy production. From 20062011, there have been 430 new energy cooperatives formed in Germany [3]. In April 2012 the REScoop 2020-20 project was launched, sponsored by the Intelligent Energy Europe programme of the European Commission. The goal of REScoop 20-20-20 is to promote the renewable energy sources cooperatives model of local citizen involvement in RES energy [4].

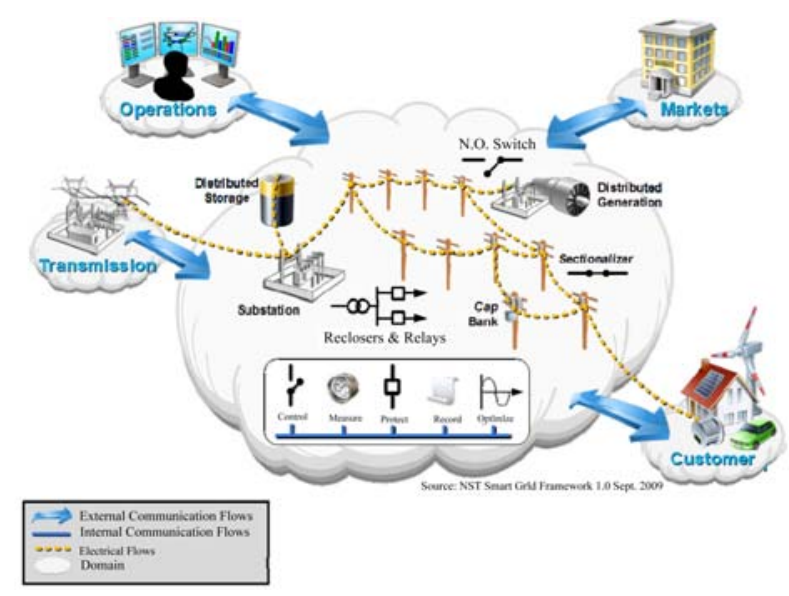

Fig. 1. Distributed Networks [1] 
In Spain there are several projects to develop rural smart grids in Catalonia (Ordis, Girona), and Castilla la Mancha y León (GE-Rural).

This paper addresses some of the technical challenges of Smart Micro-grids to its integration in the distribution network. Recent investigations on real micro-grids are reported and few rural micro-grid studies are presented to conclude the key issues to their implementation in large scale: design methodologies, load modelling and smart control strategies.

\section{Smart Micro-grids features}

The smart grid is an integration of complementary components, subsystems, and functions under the control of a intelligent and distributed command and control system. Furthermore, the growth and evolution of the smart grid is expected to come through integration of intelligent (or smart) micro-grids [5]. Keeping on mind that the application of individual distributed energy resources can cause as many problems as it may solve, the best way to manage such a system is to break the distribution system down into small clusters or microgrids, with distributed optimizing controls coordinating multi-micro-grids [6].

\section{A. Smart Micro-grid Technology}

A smart micro-grid network typically integrates the following components [7]:

- The core of micro-grids is distributed generation. Smart micro-grids are based on generators capable of meeting local demand as well as feeding the unused energy to the electricity grid. Some micro-grids are equipped with thermal power plants capable of recovering the waste heat. - A variety of loads, including residential, office industrial and agriculture loads.

- Local and distributed power-storage systems to smooth out the intermittent performance of renewable energy sources.

- Smart meters and sensors capable of measuring parameters with acceptable precision and accuracy: active power, reactive power, voltage, current, demand, etc

- Communication infrastructure that enables system components to exchange information and commands securely and reliably.

- Smart terminations and loads capable of communicating their status and accepting commands to adjust and control their performance and service level based on utility requirements.

Many of the technologies that are needed for micro-grid operation exist but are not used because of high costs.

\section{B. Smart Micro-grid Topologies}

Depending on their diversity of load, the mix of primary energy sources, and the geography and economics at work in particular areas, among other factors, micro-grids will be built with different capabilities, assets, and structures.

In both micro-grids and traditional grids, the structure of the grid has impact on the physical, technical, and operational properties of the grid. Therefore, it is important to understand the differences between grid topologies and analyse advantages and disadvantages of them:

Radial grids are based on one main line where loads and possible generation are connected in parallel. Radial networks are the most obvious choice for the base of a micro-grid rural areas. A good place for micro-grid control and protection system is at the substation

Ring grids are operated as ring and several secondary substations feed the same network. In ring grid there are two routes for power flow. Advantages of ring operation are better voltage stability and lower power losses.

Meshed grids: their main advantage is flexibility in operation but the main disadvantage is protection.

Micro-grids can be separated in four cases: island microgrid, low voltage customer micro-grid, low voltage micro-grid, and medium voltage micro-grid [8].

- In separate island micro-grid distributed generation provides electricity to one customer or small community outside the utility grid. Suitable places could be distant islands and small villages far away from the utility grid.

- Low voltage customer micro-grid operates normally parallel with utility grid. However, in case of fault in the utility grid, the micro-grid is operated as island microgrid. In this case, solar and wind power production need energy storage to offer constant electricity source.

- A low voltage micro-grid that consists of a group of low voltage customers. LV micro-grid can include anything from few consumption points to whole low voltage network fed by a MV/LV transformer. Power production of this kind of micro-grid can be based on many small generators.

- In medium voltage micro-grid, bigger production units can be applied to MV network. Medium voltage microgrids offer an opportunity for wind farms and other big production systems to produce electricity during interruption in the utility grid.

The types of distributed generation seen on rural distribution systems, includes small roof top solar units, small individual wind turbines, anaerobic digesters, landfill gas generators, biomass as well as the more traditional and still the most commonly distributed generation used today, diesel and gasoline powered stand-by type generators.

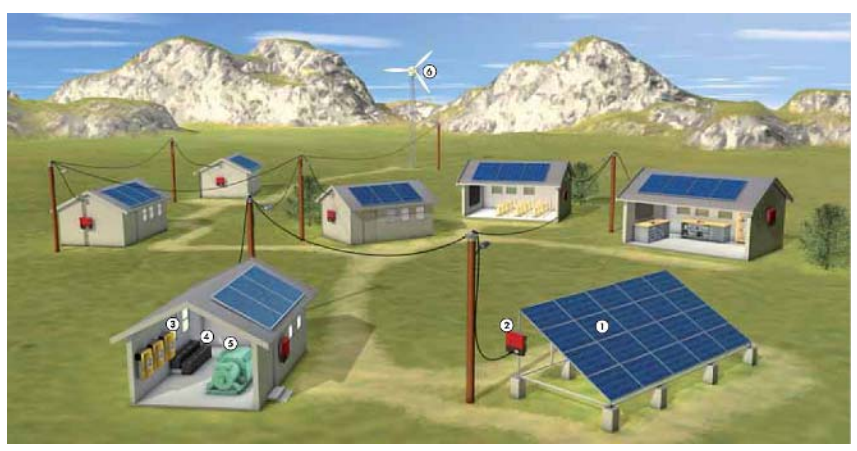

Fig. 2. Rural Distributed Generation 


\section{Integration of Smart Micro-grids into Distribution Network}

When integrating distributed generation into the distribution network, its operation is substantially changed because power flux becomes bidirectional. Distributed generators involve also an impact on feeder operation and the planning/safety practices of electric utilities. From the point of view of supply safety, they involve a review of: the design and adjustment of system protection devices; maintenance and system and restoration practices in case of faults. From the point of view of feeder operation, they may shave load curves, increase voltage values thought the feeder, reduce load levels of branches and substation transformers and reduce active and reactive losses.

Opposite to the impact that distributed generation may have at different points in the distribution network, microgrids provide the necessary flexibility to ensure the operation of the entire system as a whole, reducing the impact on the network to which they connect.

The current status of the electrical system, including the scenario that was planned for 2020, is in process of change (Strategic Research Agenda 2035) [9]. Passive distribution networks of today are in transition towards future Smart Grids. The distribution network is transforming into an instrumented, interconnected and intelligent energy distribution system. The current mind to separate tasks relevant to the operation of the transport system operator (TSO) and the operator of the distribution system (DSO) switches to a scenario in which the distribution system operation increasingly resemble the operation of the transport system and limits of each will blur. This evolution will ease the integration of micro-grids in the distribution network.

For implementing micro-grids and active distribution networks on a commercial basis, extensive research is needed, but not restricted to the following areas [10]:

- wide area active control,

- adaptive protection and control,

- network management devices,

- real-time network simulation,

- advanced sensors and measurements,

- distributed pervasive communication,

- knowledge extraction by intelligent methods and

- novel design of transmission and distribution systems.

\section{Rural Smart Grids}

Micro-grids will provide improved electric service reliability and better power quality to end customers and can also benefit local utilities by providing dispatchable load for use during peak power conditions and alleviating or postponing distribution system upgrades. There are a number of active micro-grid projects around the world involved with testing and evaluation of these advanced operating concepts for electrical distribution systems: American Micro-grids, Asian Micro-grids, European Micro-grids [11], [12]. However, there is little literature on Rural Smart Grids.
The major difference between urban and rural microgrids is that rural are connected to weak distribution networks. This means that rural micro-grids, expected to be capable of delivering big amounts of power to the distribution network, result to operate in quasi-isolated mode.

The main issues for the implementation of Rural Smart Grids are:

- Development of appropriate design methodology

- Development of new operation and planning/security practices

- Lack of knowledge in rural loads and rural distribution networks to simulation models

- Improvement on communication systems

- Design of control strategies

- Design of new protections

\section{A. Design methodology}

From the point of view of smart micro-grids design and planning, software development and field test are need to enable their large scale implementation [13].

There are two visions to design and built smart grids: first, a smart grid can be designed from scratch or, existing systems should be modified into a smart grid [14]. The second process is being carried out in rural areas where existing facilities are complemented to convert them into smart micro-grids.

Micro-grid is a complex entity which means that it is difficult to build. That is why steps towards real microgrid operation needs be planned. The conventional planning methods are designed based on electricity production in centralized power generators and delivery through passive distribution networks to end-users. The micro-grid design methodology offers a systematic approach for planning, large-scale deployment, and autonomous control of distributed generation rather than dealing with individual generation sources with diverse technologies [15].

One interesting point in the design of Rural Smart Grids is the dimensioning. On one hand, micro-grid loads must be instantaneously fed by intermittent generators, which require the implementation of storage devices to provide active power. On the other hand, simulations made show that sensitivity for voltage fluctuations gets higher in weaker grids and the control system needs to be more accurate and stabile. Thus, one of the challenges of the control in weak grids is the need of reactive power to control the voltage but if reactive power is transferred in the grid, it causes losses. All these reasons must be kept on mind when dimensioning micro-grid components [8].

In this line, Phrakonkham et al. propose a combination of simulation, design and optimization tools within open architecture software such as Matlab/Simulink to cope with the multi-objective optimization and the micro-grid configuration of a standalone renewable hybrid system [16]. 


\section{B. Rural loads characterization for micro-grid modelling}

Micro-grids include a wide variety of generator technologies and storage devices mostly coupled via power electronic interfaces. Modelling of these components in steady-state and transient conditions is far from trivial, so they have been highly studied. However, there are not many choices for load models. It has been recognized that the load model has a significant influence on power system digital simulation, especially on the dynamic simulation. Inappropriate or inaccurate load models may cause unreliable results in the power flow calculation, the short-circuit calculation and stability analysis. It can further affect the power system operation, control and design.

There are generally two types of load models; the static load model and the dynamic load model. The most used static load models are the polynomial load model and the exponential load model. The most popular dynamic load model is the induction motor model. As a matter of fact, the induction motors could consume $60 \%-70 \%$ of the total energy supply of a power grid [17].

Micro-grids loads are analyzed by means of two modeling approaches: component-based approach and measurementbased approach. Podmore and Robinson implement component-based models to the simulation and control of loads [13]. However, Rudion et al. show that load model accuracy could be improved by using the measurementbased approach, so that the load model could better depict the static and dynamic performance of the real system load. [17]. Unfortunately, little data is available in developing countries of rural feeder load as well as the habits of rural villagers, leading to significant gaps in the required database [18].

Another characteristic of micro-grids is that they may use single-phase circuits and be loaded with single-phase loads. These factors generate unbalanced conditions that can be accentuated with the interaction of dynamic loads such as induction motors. To model these effects, a suitable analytical tool has been developed that represents the system with its three phases, the neutral conductors, the ground conductors and the connections to ground and includes models of various micro-sources [19].

However, the ability to accurately model distribution systems with smart grid components and associated behaviors will require distribution system analysis tools to evolve significantly [20].

Finally, there is still a lack of knowledge on characterizing which are the necessary feeder operating variables to assess the distributed generators impact on different feeders. For the moment, in default of rural distribution network benchmarks, several authors use modified IEEE PES Distribution Test Feeders [21] to analyze the control strategies and operation solutions proposed in their studies [22], [23].

\section{Smart Control Strategies}

The control system of a micro-grid is designed to safely operate the system when it is connected to the grid or in stand-alone mode. This system may be based on a central controller or embedded as autonomous parts of each distributed generator.

Micro-grid controllers must ensure that [24]:

- micro-sources work properly at predefined operating point or slightly different from the predefined operating point but still satisfy the operating limits;

- $\quad$ active and reactive powers are transferred according to necessity of the micro-grids and/or the distribution system;

- disconnection and reconnection processes are conducted seamlessly;

- $\quad$ in case of general failure, the micro-grid is able to operate through black-start; and

- $\quad$ energy storage systems can support the micro-grid

Control of the micro-grid is totally different to what distributed generation units are used to do. When the micro-grid is disconnected from the grid, control system must control the local voltage and frequency and provide the instantaneous active and reactive power differences between generators and loads.

When the micro-grid is in stand-alone mode, frequency control is the most important problem. The frequency response of conventional systems is based on rotating masses and these are considered as essential for the stability of the systems. In contrast, micro-grids are inherently converter-dominated grids with very little directly connected rotating masse. Thus, the electronic converter control systems must be adapted to provide the response previously obtained from directly connected rotating masses. The frequency control strategy of microgrids should coordinate the capabilities of the microsources to change their active power (through frequency control droops), the response of the storage systems, and load shedding.

Voltage regulation is considered the second main objective of networks to guarantee their local reliability and stability. It has been observed that systems with high penetration of distributed generation experience voltage and/or reactive power changes and oscillations in the connection point. Local voltage control must be designed to avoid these changes and the circulation of reactive currents between sources [25]. The capability of modern power electronic interfaces offers solutions to the provision of reactive power locally by the adoption of a voltage versus reactive current droop controller, similar to the droop controller for frequency control [5].

One of the most important problems of rural smart grid control is the nature of load characteristics, like load diversity and time variability. To cope with this problem, the control system must have a hierarchical structure. 
The control level of hierarchical systems can be classified on [26]:

- local controllers consisting of Micro-source Controllers (MCs) and Load Controllers (LCs);

- Micro-grid Central Controllers (MGCCs); and

- Distribution Management System (DMS).

Local controls are the basis of micro-grid controls and their main purpose is to control micro-sources. These controllers are intended to control operating points of the micro-sources as well as their power-electronic interfaces, based on local voltages and currents measured data.

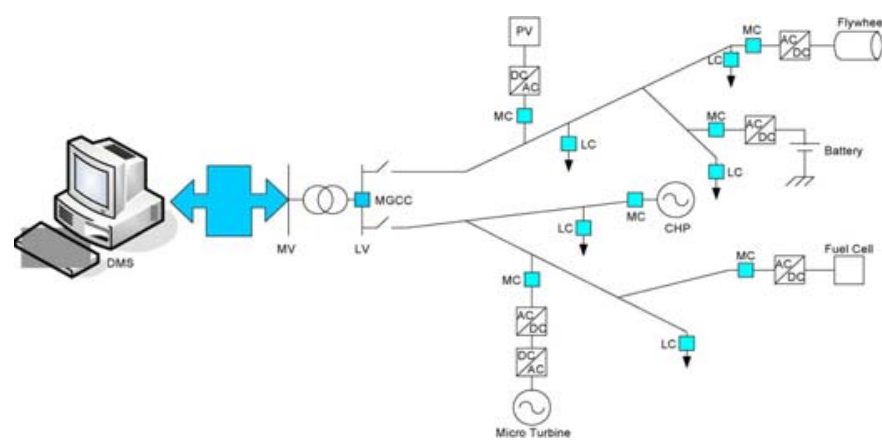

Fig. 2. Hierarchical control of micro-grid.[26]

On the other hand, the ability to directly control large amounts of customer loads will provide an important improvement to the operation of smart micro-grids. Direct load controls can be used to enhance transient angle stability, frequency stability, voltage (transient collapse, midterm or classical voltage collapse, long-term voltage collapse), and thermal overload [13].

Incorporating all these control technologies rural microgrids are evolving towards rural smart grids: Smallwood and J. Wennermark show the benefits of implementing a SCADA system in the rural distribution network [27].

In [28], control strategies and coordination of centralized and decentralized frequency control of distributed energy resources are illustrated in a detailed isolated rural microgrid model. This paper shows that good control strategies applied to diesel generators, storage and distributed generation can achieve satisfactory control performance in frequency deviations and also allow the minimal size of storage corresponding to a certain wind penetration level.

Finally, advanced control techniques like the artificial intelligence and multi agents are applied to build a microgrid system, comprised of an agent system which is not only hybrid, centralized, but also decentralized with easily deployable wind-turbines for generating electricity [29].

\section{$5 \quad$ Conclusion}

Micro-grids are a key point for integrating distributed generation in the network, and allow transition of today's passive distribution networks to smart grids. The rural environment is suitable for such applications, for having renewable resources and can be economically justifiable.
This paper addresses some of the key technical challenges that are needed to overcome to achieve the smart grids of the future integrating Rural Smart Microgrids.

However, for this purpose rural smart micro-grids must be improved in design, simulation, protection, controllability and communication resources. In this line, there is still a lot of work to do in loads characterization, modeling of rural distribution networks, control of microsources and customer loads, as well as in the development of benchmarks for rural grids.

\section{References}

[1] N.A. Hidayatullah, B. Stojcevski and A. Kalam, “Analysis of Distributed Generation Systems , Smart Grid Technologies and Future Motivators Influencing Change in the Electricity Sector”, Smart Grid and Renewable Energy (2011) Vol. 2, pp. 216-229.

[2] SmartGrids: European Technology Platform. Disponible en http://www.smartgrids.eu.

[3] A. Bilek, "Revitalizing Rural Communities through the Renewable Energy Cooperative", This paper is part three of a six-part series on the German Energy Transition (Energiewende). Published by the Heinrich Böll Stiftung, Washington, D.C., (2012)

[4] http://www.rescoop.eu/about-rescoop-20-20-20

[5] H. Farhangi, "The path of the smart grid", in IEEE Power and Energy Magazine January-February 2010, Vol. 8 (1), pp. 18-28.

[6] R. H. Lasseter, "Smart Distribution: Coupled Microgrids", in Proc. IEEE 2011, Vol. 99 (6), pp. 1074-1082.

[7] R. Saint, "Rural Distribution System Planning using Smart Grid Technologies”, in Proc. IEEE Rural Electric Power Conference, REPC '09. B3-8

[8] H. Laaksonen, Technical solutions for low-voltage microgrid concept, Ph.D. dissertation, University of. Vaasa Finland (2011).

[9] Strategic Research Agenda Smart Grids 2035. SmartGrids: European Technology Platform

[10] S. Chowdhury, S.P. Chowdhury and P. Crossley, "Microgrid and Active Distribution Network", The Institution of Engineering and Technology, London, United Kingdom (2009).

[11] M. Barnes, G.Ventakaramanan, J.Kondoh, R. Lasseter, et al, "Real-World MicroGrids- An Overview", in Proc. IEEE International Conference System of Systems Engineering, 2007. SoSE '07. pp. 1-8.

[12] M. Shamshiri, Ch.K. Gan, Ch,W. Tan, “A Review of Recent Development in Smart Grid and Micro-Grid Laboratories”, in Proc. IEEE International Power Engineering and Optimization Conference PEOCO2012, Melaka, Malaysia, 6-7 June 2012. pp. 367-372.

[13] R. Podmore and MR Robinson "The Role of Simulators for Smart Grid Development” IEEE TRANSACTIONS ON SMART GRID, 2010, Vol. 1 (2), pp 205 - 212.

[14] R. Hassan and G. Radman, "Survey on Smart Grid" Proceedings of IEEE SoutheastCon 2010 (SoutheastCon 2010) pp. $210-213$.

[15] A. Brissette, A. Hoke, D. Maksimovic, A. Pratt, A. "A microgrid modeling and simulation platform for system evaluation on a range of time scales" in Proc. IEEE Energy Conversion Congress and Exposition (ECCE), 2011 pp. 968 976.

[16] S. Phrakonkham, J.Y. Le Cenadec, D. Diallo et al. "Reviews on Micro-Grid configuration and dedicated Hybrid 
System Optimization Software Tools: Application to Laos”, Engineering Journal 2010, Vol, 14 (3).

[17] K. Rudion, H. Guo, H. Abildgaard, Z. A. Styczynski, "Nonlinear load modeling - Requirements and preparation for measurement” IEEE Power \& Energy Society General Meeting, 2009. PES '09.

[18] J.C. Hernández, A. Medina, and F. Jurado, "Impact comparison of PV system integration into rural and urban feeders”, Energy Conversion and Management, Elsevier 2008 Vol. 49, 2008 pp. 1747-1765.

[19] Hatziargyriou, "Modelling and simulation of Microgrids", First International Conference on "Integration of RES and DER", Brussels, 1-3 December 2004.

[20] R. F. Arritt and R. C. Dugan, "Distribution System Analysis and the Future Smart Grid", IEEE Transactions on Industry Applications 2011, Vol. 47 (6), pp. 2343-2350.

[21] IEEE PES, Distribution Test Feeders, [Online]. Available: http://www.ewh.ieee.org/soc/pes/dsacom/testfeeders/index.html. [22] G. N. Kariniotakis, N. L. Soultanis, A. I. Tsouchnikas, S. A. Papathanasiou, and N. D. Hatziargyriou, "Dynamic modeling of microgrids", in Proc. International Conference on Future Power Systems, 2005, pp. 7

[23] L. Ye, H. B. Sun, X. R. Song, and L. C. Li, "Dynamic modeling of a hybrid wind/solar/hydro microgrid in EMTP/ATP”, Renewable Energy, 2012 Vol. 39, pp. 96-106.
[24] R. Zamora and A.K. Srivastava, "Controls for microgrids with storage: Review, challenges, and research needs", Renewable and Sustainable Energy Reviews, (2010) Vol. 14, pp. 2009-2018.

[25] D.M. Farmer, P.B. Ingle, and S.H. Morgan, "Integrated Volt/VAR Control on Rural Distribution Systems", in Proc. IEEE RURAL ELECTRIC POWER CONFERENCE (REPC) 2011.

[26] AG. Tsikalakis and N.D. Hatziargyriou, "Centralized Control for Optimizing Microgrids Operation”, IEEE TRANSACTIONS ON ENERGY CONVERSION, VOL. 23 (1), 2008 pp. 241-247.

[27] C.L. Smallwood and J. Wennermark, "The Benefits of Distribution Automation to a Rural Electric Cooperative", in Proc. 53rd Annual IEEE Rural Electric Power Conference 2009.

[28] S. Lu, M.A. Elizondo, N. Samaan et al, "Resources to Maximize the Use of Wind Power in Rural Microgrids", in Proc. IEEE Power and Energy Society General Meeting PESGM 2011

[29] B-Ch. Min, H. Chaudhry, E.T. Matson, Eric T.; et al, "Rural Energy Security Using Autonomous Micro-turbine Smart Grids”, in Proc. IEEE RURAL ELECTRIC POWER CONFERENCE (REPC) 2011. 